\title{
Register nominum ad Vol XXXIII
}

Abderhalden, B., 33, 396 (B) Adlersberg, L., v. Iliesco, C.

Bachmann, K., v. Bar, C. G. Bajusz, E., v. Selye, H. Bar, C. G., und Bachmann, K., 33, 435

Bayley, B. H., 33, 395 (B) Benyamine, B., v. Jouve, A. Blumberger, K., v. Weber, A. Bompiani, G. D., v. Farulla, A. Brandenburg, P., und Wick, E.,

35, 121 Bühlmann, A., v. Rossier, P. H. Bülow, K., 33, 211 Busch, W., und Eiselsberg, K., 33, 137

Carlier, J., et Lejeune-Ledant, G.,

33, 224 Code, C. F., Creamer, B., Schlegel,

J. F, 33, 451 (B) Corriol, J., v. Jouve, A. Creamer, B., v. Code, C. F.

Demetresco, L., v. Iliesco, C.

Edwards, W. S., 33, 158 (B) Efraim, M., v. Iliesco, C. Eiselsberg, K., v. Busch, W. Eliasch, H., v. Werkö, L.

Fabrizi, G., v. Perri, T. di Farulla, A., Sangiorgi, M., and

Bompiani, G. D., 33, 269 Faucon, N., 33, 331 (B)

Gillmann, H., 33, 21 (S) -, and Varsos, A., 33, 230 Giraud, G., 33, 450 (B) Greensher, J., v.

Widmer, L. K. Gríbbe, P., Lind, J., Linko, E. and Wegelius, C, 33, 293

Hegglin, B., v. Isler, U. Heinecker, R. und Zipf, K. E.,

33, 407 Holzmann, M., und Schaub, F.,

33, 84 (S) Honetz, N., Hueber, E. F., und

Scheid, H., 33, 333 Hueber, E. F., v. Honetz, N. Hupka, K., und Wenger, B., 33, 259 Hürlimann, A., v. Imhof, P.

ad Vol. XXXIII

Iliesco, C, Savulesco, V., Adlersberg, L., Efraim, M., et Demetresco, L., 33, 152

Imhof, P., und Hürlimann, A., 33, 2 (S)

Isler, U., und Hegglin, B., 33, 69 (S)

Jacobi, J., und Loeweneck, M.,

33, 332 (B) Jourdan, P. F., 33, 330 (B) Jouve, A., Corriol, J., Velasque, P.,

Benyamine, B., et Peytavy, R.,

33, $45(\mathrm{~S})$

Kajas, S., v. Linko, E. Kaufmann-Staemmler, 33, 331 (B) Klepzig, H., v. Beindell, H. Köberle, F., 33, 384 Köhn, K., und Bichter, M., 33,451 (B)

Lamb, L. E., 33, 394 (B) Landes, G., 33, 450 (B) Langen, C. D. de, 33, 249 Lejeune-Ledant, G., v. Carlier, J. Lind, J., v. Gribbe, P. Linko, E., and Kajas, S., 33, 313 -, v. Gribbe, P. Loeweneck, M., v. Jacobi, J.

Mannheimer, E.,

v. Müller-Brunotte, P. Marriott, H. J. L, 33, 159 (B) Müller-Brunotte, P., und Mannheimer, E., 33, 371

Neoral, L., v. Wiedermann, B.

Partilla, H, 33, 108 
Paula, e Silva, P. de, Raab, W.,

Starcheska, Y.K., 33, 365 -, v. Raab, W. Pauli, H., 33, 63 (S) Perri, T. di, and Fabrizi, G., 33, 97

Peytavy, R., v. Jouve, A. Plotz, M., 33, 330 (B)

Raab, W., de Paula e Silva, P., and

Starcheska, Y.K., 33, 350 -, v. Paula e Silva, P. de Beindell, H., und Klepzig, H.,

33, 449 (B) Richter, M., v. Köhn, K.

456 Register

Romano, D. jr., 33, 241 Rossier, P. H., Bühlmann, A., und Wiesinger, K., 33, 332 (B)

Sanabria, A., and Soto, R., 33, 130 Sangiorgi, M., v. Farulla, A. Savulesco, V., v. Iliesco, C.

Schaub, F., v. Holzmann, M. Scheid, H. v. Honetz, N. Schlegel, J. F., v. Code, C. F. Schmid, A., v. Wüthrich, F. Schütz, E, 33, 396 (B) Schweizer, W, 33, 32 (S), 40 (S) Selye, H,, and Bajusz, E., 33, 305 Söderström, N., 33, 397 Soldati, L. de, 33, 160 (B) Soto, It., v. Sanabria, A. Spang, K., 33, 268 (B) Spirig, P., v. Wüthrich, F. Starcheska, Y. K., v. Paula e Silva P. de -, Raab, W. Strausak, A., v. Wüthrich, F.

Teschendorf, W., 33, 330 (B) Thomasson, ß., v. Werkö, L. Trethewie, E. R., 33, 423

i ad Vol. XXXIII

Van den Berg, J. W., v.

Vries, H. K. de Varnauskas, E., v. Werkö, L. Varsos, A., v. Gillmann, H. P. Velasque, P., v.

Jouve, A. Vries, H. K. de, and van den Berg,

J. W., 33, 195

Weber, A., und Blumberger, K.

33, 396 (B) Wegelius, C, v. Gribbe, P. Wenger, R., v. Hupka, K. Werkö, L., Eliasch, H., Thomasson,

B., and Varnauskas, E., 33, 161 Wick, E., v. Brandenburg, P. Widmer, L. K., und Greensher, J. 33, 415 Wiedermann, B., Neoral, L., und

Wiedermann, M., 33, 323 Wiedermann, M., v. Wiedermann, B. Wiesinger, K., v. Rossier, P. H. Wirecki, M., 33 suppl., 1 Wüthrich, F., Schmid, A., Spirig, P.,

und Strausak, A., 33, 11 (S)

Zipf, K. E., v. Heinecker, R. 